Individualizing Evidence-Based Treatment of Neuropsychiatrically Complex

Patients: Process-Based Targets For Change in Parkinson's Disease

S.L. Mann, R. Miller, L. St. Hill, \& R.D. Dobkin

Pragmatic Case Studies in Psychotherapy, http://pcsp.libraries.rutgers.edu

Volume 16, Module 1, Article 2, pp. 104-117, 07-25-20 [copyright by authors]

Commentary on: Telephone-Based, Clinician-Guided Self-Help Cognitive

Behavioral Therapy for Depression in Parkinson's Disease (dPD): The Responder Cases of "Alice" and "Carl," and the Nonresponder Cases of "Ethan" and "Gary"

\title{
Individualizing Evidence-Based Treatment of Neuropsychiatrically Complex Patients: Process-Based Targets for Change in Parkinson's Depression
}

\author{
SARAH L. MANN, ${ }^{\mathrm{a}}$ RACHAEL MILLER, ${ }^{\mathrm{a}}$ \\ LAUREN ST. HILL, ${ }^{a}$ \& ROSEANNE D. DOBKIN ${ }^{b, c}$
}

${ }^{a}$ VA New Jersey Health Care System, Lyons, NJ

${ }^{\mathrm{b}}$ Rutgers-Robert Wood Johnson Medical School, Piscataway, NJ

${ }^{\mathrm{c}}$ Correspondence regarding this article should be sent to Roseanne Dobkin, Rutgers-Robert Wood Johnson Medical School, 671 Hoes Lane, D-317, Piscataway, NJ 08854

Email: dobkinro@rutgers.edu

\begin{abstract}
Providing evidence-based treatment always requires responding in the moment to apparent gaps between the protocol and the patient's presenting needs and preferences. In the treatment of depression in Parkinson's disease (PD), research has shown that providing PD-specialized, empirically supported interventions is paramount. However, given PD's highly heterogeneous symptom presentations, adapting and individualizing treatment to address each patient's unique constellation of neuropsychiatric symptoms and PD-related physical challenges is equally important. This Commentary on the article by Dr. Logan Durland (2020) focuses on the importance of attending to process-based factors to inform protocol adaptations in the treatment of Parkinson's depression, guided by the framework of functional analytic psychotherapy (FAP). The FAP approach applies behavioral principles to in-session processes as a means of highlighting and therapeutically targeting clinically relevant behaviors in real time. Seeking such opportunities to foster within-session change may be especially important when providing evidence-based treatment to individuals with co-occurring mental health issues and chronic, functionally limiting medical problems like PD. As these patients manage the interactions between the complex demands of illness self-management and the burden of mental health symptoms, they must respond adaptively to unpredictable daily challenges. Harnessing moments of clinically relevant struggle during sessions in order to support and reinforce new responsesincluding new ways of approaching the learning process itself — can help patients consolidate both the coping skills themselves and flexibility and confidence to apply them.
\end{abstract}

Key Words: depression; Parkinson's disease (PD); depression in Parkinson's disease (dPD); cognitivebehavioral therapy; evidence-based practice; Functional Analytic Psychotherapy; case study; clinical case study 
Individualizing Evidence-Based Treatment of Neuropsychiatrically Complex

Patients: Process-Based Targets For Change in Parkinson's Disease

S.L. Mann, R. Miller, L. St. Hill, \& R.D. Dobkin

Pragmatic Case Studies in Psychotherapy, http://pcsp.libraries.rutgers.edu

Volume 16, Module 1, Article 2, pp. 104-117, 07-25-20 [copyright by authors]

\section{INTRODUCTION}

At the forefront of Parkinson's disease (PD) care, the need for individualized, patientcentered disease management is gaining prominence (Titova \& Chaudhuri, 2017). This change reflects the growing recognition that while $\mathrm{PD}$ is defined by a triad of motor symptoms - tremor, bradykinesia (slowed movement), and muscle stiffness or rigidity - it also involves a variety of neuropsychiatric symptoms which are, overall, even more detrimental to patients' quality of life (Hinnell et al., 2012). Depression, PD's most prevalent psychological concern (Weintraub \& Mamikonyan, 2019), is also the single strongest predictor of caregiver burden and of patients' distress and disability (Forsaa, Larsen, Wentzel-Larsen, Herlofson, \& Alves, 2008; MartínezMartín et al., 2007).

The need for personalized Parkinson's management extends to PD-specialized mental health care (Pontone et al., 2019). Within this domain, increasing access to treatment for depression in PD (dPD) is an urgent priority, due to depression's severe, deleterious effects on overall PD outcomes. Unlike traditional depression treatments, PD-informed cognitive-behavior therapy (CBT) addresses depression and PD management simultaneously, applying both a PD knowledge base and expertise in the behavioral and cognitive interactions between depression and PD symptoms. Given numerous treatment access barriers, including a marked shortage of mental health clinicians with adequate knowledge of a movement disorder like PD, development of an evidence-based, PD-informed, telehealth-deliverable protocol (Dobkin et al., 2020a; Dobkin et al., 2020b) is key to meeting a large and growing depression treatment need in the Parkinson's community. However, the urgency of meeting this need with a widely disseminable protocol must be balanced against the need to adapt treatment to patients' considerably varied experiences with PD and depression.

This tension between fidelity and flexibility in $\mathrm{APD}$ treatment reflects broader questions in the field of evidence-based psychotherapy in general, regarding the relationship of a given treatment protocol to the therapeutic process that unfolds between an individual provider and patient. By definition, evidence-based practice involves treatment adaptation, to integrate "the best available research with clinical expertise in the context of patient characteristics, culture, and preferences"(American Psychological Association, 2005). Yet, specific questions that arise during sessions about whether and how to modify treatment from the generic treatment manual to meet an individual patient's needs often hinge on the underspecified variable of clinical judgment. How can providers respond in the moment to apparent gaps between the patient's presenting needs and preferences and the protocol as written?

As one approach to answering this question, Functional Analytic Psychotherapy (FAP) offers a set of behavioral principles for conceptualizing and responding to such challenges, which can be applied as a "framework for implementing evidence-based practices" (Holman et al., 2012). FAP emphasizes the ways in which principles of behaviorism operate during sessions and within the therapeutic alliance (Kanter et al., 2017). By focusing attention on in-session processes and interactions, and considering how they may reflect the patient's presenting problems, FAP highlights "in-the-moment," accessible behavioral targets and mechanisms for 
Individualizing Evidence-Based Treatment of Neuropsychiatrically Complex

Patients: Process-Based Targets For Change in Parkinson's Disease

S.L. Mann, R. Miller, L. St. Hill, \& R.D. Dobkin

Pragmatic Case Studies in Psychotherapy, http://pcsp.libraries.rutgers.edu

Volume 16, Module 1, Article 2, pp. 104-117, 07-25-20 [copyright by authors]

therapeutic change, as well as ways of supporting generalization outside of sessions. Applying this lens to the therapeutic process can help the therapist to notice and respond to clinically relevant in-session behaviors and reinforce change (Kanter et al., 2017).

FAP proponents highlight at least two major benefits of integrating this approach into protocol-guided, evidence-based psychotherapy (EBP; Hopko \& Hopko, 1999). For one, FAP directly mobilizes the therapeutic alliance in support of behavior change. The alliance is generally acknowledged among nonspecific contributors to all therapy outcomes, but it receives less explicit attention in many evidence-based protocols (Laska, Gurman, \& Wampold, 2014). Second, by utilizing behavioral principles within sessions, FAP can maximize their power, as the therapist can provide immediate reinforcement and can promote generalization by making explicit the functional parallels between in-session and daily-life behavior change (Kanter et al., 2017). In the literature, FAP has most frequently been incorporated into evidence-based, behavioral treatments for depression (e.g., cognitive-behavioral therapy, behavioral activation), and has shown support for added benefit over the same intervention delivered without FAP integration (Kanter et al., 2017).

FAP's emphasis on engaging in-session behaviors as targets for change may be especially valuable for delivering manualized CBT for depression in Parkinson's disease, because in addition to the patient's history and strengths, every session is shaped by the patient's specific constellation of cognitive, emotional, and behavioral PD symptoms. In many cases, functional limitations that become clear only session-by-session come to define the unique gaps the clinician must bridge between each individual with $\mathrm{dPD}$ and the treatment protocol, to individually tailor an effort-rewarding treatment experience. Recognizing parallels, as FAP suggests, between the patient's reported daily struggles (i.e., with depression and PD) and the inevitable challenges that arise during sessions, opens the possibility of using in-session difficulties as collaborative instances of inquiry and observation, and ideally, as opportunities for carefully supported, celebrated change.

The four complex cases Durland (2020) discusses each include his skillful adaptations of the protocol to make treatment accessible and meaningful to the patient. In each one, Durland also faces patients' in-session difficulties engaging with the protocol for a variety of reasons, including anxiety about public speaking, social activities, and stamina; care-partner dynamics; cognitive difficulties, and struggles against the PD diagnosis itself. This commentary examines these process-based difficulties with an FAP-guided focus - that is, seeking opportunities to view these in-session challenges as manifestations of, or clues about, the patient's broader presenting problems, and to adapt treatment accordingly_aiming to directly engage difficult, clinically relevant in-session behaviors to support out-of-session change.

\section{ALICE, A RESPONDER, AND BOB: SESSION PARTICIPATION AS BEHAVIORAL ACTIVATION}

In Alice's case, Durland worked carefully with Alice and her husband, Bob, to adapt the therapy protocol's activity scheduling exercise to target Alice's initial reticence in her own 
Individualizing Evidence-Based Treatment of Neuropsychiatrically Complex

Patients: Process-Based Targets For Change in Parkinson's Disease

S.L. Mann, R. Miller, L. St. Hill, \& R.D. Dobkin

Pragmatic Case Studies in Psychotherapy, http://pcsp.libraries.rutgers.edu

Volume 16, Module 1, Article 2, pp. 104-117, 07-25-20 [copyright by authors]

sessions. In the first session, Bob served as the couple's "spokesperson" while Alice's remained largely silent. In this dynamic, Durland observed a real-time effect of Alice and Bob's shared belief in the severity and consequences of Alice's functional limitations. That is, Alice relied on Bob across many domains, rarely expressing her own needs, because the couple presumed that her PD symptoms largely prevented independent functioning. As the couple grew willing to carefully test their shared belief and the scope of Alice's limitations, using purposeful activity scheduling, Durland astutely offered the sessions themselves as one of Alice's testing grounds for behavior change. In other words, despite concerns that it might overtax Alice, Alice and Bob agreed to "see how Alice handled taking on more of the speaking role." This intervention saw her participation increase to roughly equal Bob's in the subsequent session.

To address the couple's worries about this increased demand on Alice, Durland engaged in sensitive, collaborative planning for a flexible in-session response if Alice should express a need to slow down or take a break. In doing so, he modeled a new approach for the couple to activities "at the outer limits of Alice's perceived abilities." Rather than assuming Alice should not attempt independent activities to avoid becoming overwhelmed, they could consider safe, incremental ways for her to become increasingly active, backstopped by a shared plan to accommodate her potential need for a break or on-the-spot activity modification.

Drawing Alice into speaking throughout her sessions also provided opportunities for realtime reinforcement that strengthened important changes in the couple's communication. For one, Durland focused on conversing directly with Alice, modeling patience with her pace of speech and curiosity in contributions, which reinforced her for persisting rather than deferring to Bob. Second, their plan for her to speak more in sessions included an agreement about adaptive responding if Alice expressed a need to rest or slow the pace. This plan realigned the group to take cues about Alice's experience and limitations from her as those needs arose. In this new dynamic, the group - including Alice - invested in her self-efficacy to be her own "spokesperson" and stood ready provide swift reinforcement for her initiative, in the form of accommodating her expressed needs, if she made a request. Durland also reinforced several changes in Bob's communication, recognizing his restraint when he previously would have "rescued" Alice from a speaking difficulty, his openness to understanding her experiences even when they upended his expectations, and his supportive feedback appropriately offered. These interventions skillfully shaped Bob's role in sessions from doing for Alice to supporting her therapeutic accomplishments - paralleling his broader shift at home from over-functioning on her behalf to helping her reach her own optimal capacity.

Durland notes Alice's many successes as she resumes more independent activities. One additional opportunity to build on these changes and bolster her independence might have involved gauging her willingness to participate in some individual sessions, independently from Bob. (To maintain rapport and engagement with Bob and afford him the opportunity to address individual challenges in his caregiver role transition, he could have been offered at least one individual care-partner coaching session as well.) For Alice, these sessions could have provided another low-risk opportunity to exceed her prior expectations of herself, shoring up her 
Individualizing Evidence-Based Treatment of Neuropsychiatrically Complex

Patients: Process-Based Targets For Change in Parkinson's Disease

S.L. Mann, R. Miller, L. St. Hill, \& R.D. Dobkin

Pragmatic Case Studies in Psychotherapy, http://pcsp.libraries.rutgers.edu

Volume 16, Module 1, Article 2, pp. 104-117, 07-25-20 [copyright by authors]

confidence in this domain and perhaps encouraging her to generalize her reclaimed independence in her social life and in therapy to interactions with her other healthcare providers.

Individual sessions also might have provided a forum for directly addressing Alice's negative thoughts. One approach could have involved using Alice's successes in behavioral activation to highlight behavior-thought connections, via the role her new behaviors played in changing her thoughts. As one example, her worries about experiencing PD symptoms in front of her friends reportedly decreased following positive social experiences; this cognitive change then reinforced additional outgoing behavior. This example might have provided Alice with a concrete, experiential illustration of the ways thoughts, behaviors, and biological factors (in this instance, neurochemical and structural and functional brain changes triggered by PD) can work together to maintain depression - and that intervening at any of these points can improve mood. Using this example to teach her some simple cognitive restructuring techniques (e.g., What is the best, worst, and most likely possible outcome?), might have provided a valuable experience with flexible problem solving, demonstrating that there may be alternative routes to a goal (i.e., reframing), further supporting her perceptions of empowerment.

Individual sessions with Alice also might have provided a forum for exploring thoughts and feelings she found difficult to share with Bob. Given Alice's emotional sensitivity, previously unarticulated shame about her PD symptoms, and prior over-reliance on Bob, she might have believed herself to be a burden to him, which is a common concern in dPD. If so, voicing this painful thought might have been easier for Alice in a private session. Similarly, she might have benefitted from the opportunity to examine the basis for the thought and work through restructuring it for herself with Durland's help, without feeling obliged to respond to the effects of this potent, painful thought on Bob.

\section{ETHAN, A NONRESPONDER, AND FAY: DISENTANGLING CAREGIVER DYNAMICS FROM COGNITIVE DIFFICULTIES}

The effect of Bob's participation in Alice's treatment was largely positive, whereas Faye's attendance in Ethan's sessions brought many challenges, even detriments, to the process. Durland viewed Fay's participation as necessary, largely to help compensate for Ethan's significant cognitive and speech difficulties. These deficits, respectively, impaired Ethan's ability to comprehend and retain program material and to express himself clearly to Durland. In contrast, Fay could grasp and apply the program concepts and "translate" for Ethan in sessions.

Yet, as Durland highlights, in her intermediary role, Fay presents some program material to Ethan in a manner that accurately reflects key content (e.g., providing relevant evidence against a negative belief of his that was impeding behavioral activation) - still taking a hostile, critical tone in doing this. This dynamic seemed to sideline Ethan somewhat within his own treatment, leaving him and Durland both reliant on Fay. As Ethan clearly despaired about feeling "useless" to his loved ones, this pattern risked reinforcing some of the interpersonal dynamics and related beliefs that were likely contributing to Ethan's depression. Ethan's lack of self- 
Individualizing Evidence-Based Treatment of Neuropsychiatrically Complex

Patients: Process-Based Targets For Change in Parkinson's Disease

S.L. Mann, R. Miller, L. St. Hill, \& R.D. Dobkin

Pragmatic Case Studies in Psychotherapy, http://pcsp.libraries.rutgers.edu

Volume 16, Module 1, Article 2, pp. 104-117, 07-25-20 [copyright by authors]

efficacy and Fay's apparent frustration with the intense demands of his significant impairments, both presented significant challenges in these sessions.

This pattern emerged early, at a point when Durland "saw Fay's participation as crucial" and worried that he "might alienate her if [he] suggested that her communication style was unproductive" (p. 60). Durland therefore conceptualizes his own challenge as "maximizing Fay's positive contributions while limiting her negative impact" (p. 59). This choice makes sense in light of Durland's felt obligation, within a treatment trial, to maximize protocol adherence, as the material seemed inaccessible to Ethan without Fay's help - and even with her involvement, potentially outstripped Ethan's capabilities. Prioritizing adherence therefore may have steered difficult treatment decisions toward retaining and "capitalizing on" Fay's double-edged contributions, despite their potentially negative effects on Ethan.

An alternative approach could have involved working with Ethan and Fay separately, at least early in treatment, allowing Durland to assess their very disparate capabilities, needs, and perspectives, as well as their distinct roles in maintaining Ethan's depression. Though holding individual sessions with Ethan would have posed new communicative challenges, it might have provided Durland a clearer picture of Ethan's functional capacities, apart from his tendency to defer to Fay or withdraw from her criticism. Information gleaned from these sessions, including the feasibility of developing independent rapport with Ethan by phone, might have informed substantial treatment modifications to make carefully chosen program components as accessible and meaningful as possible for him. In Ethan's case, this individualized approach would have likely required drastically reducing cognitive and functional demands by limiting and simplifying the treatment material presented and narrowing initial treatment goals.

The rationale for making such adjustments, in consultation with Durland's supervisor, Dr. Dobkin, would have been not only to make the content accessible but also to create a treatment process that could reinforce Ethan's early efforts by maximizing his engagement and success. For example, rather than attempting to consolidate new skills quickly "since Ethan's difficulties with working memory often left a narrow window of opportunity" (p. 60), another approach might have involved selecting fewer skills to teach, and allocating more time to each one, including lots of in-session repetition and practice. In this approach, the treatment modules could be described as a "buffet" of coping skills, with the therapist and client collaborating to choose those most likely to be helpful, rather than attempting cover the whole curriculum. Activity scheduling could have begun with very low-demand changes that nonetheless provided Ethan with new evidence for his initiative and self-efficacy. Cognitive restructuring could have been concretized by exploring in conversation, "What would you tell a loved one facing this kind of situation?," providing reinforcement through reflective listening, rather than treating it as written restructuring exercise. Relaxation practice could have been simplified as a gentle breathing exercise to rehearse in session ("smell the rose, blow out the candle"), and then generalized to falling asleep at night. To allow each session to build, however slowly and incrementally, on those prior, it might have been helpful to send Ethan simple session notes after each meeting. For example, these could have taken the form of a brief, large-type, bulleted list using simple language to review key points discussed and to praise Ethan's helpful contributions. 
Individualizing Evidence-Based Treatment of Neuropsychiatrically Complex

Patients: Process-Based Targets For Change in Parkinson's Disease

S.L. Mann, R. Miller, L. St. Hill, \& R.D. Dobkin

Pragmatic Case Studies in Psychotherapy, http://pcsp.libraries.rutgers.edu

Volume 16, Module 1, Article 2, pp. 104-117, 07-25-20 [copyright by authors]

Given Ethan's difficulties with homework despite receiving email reminders, it might have been possible for Ethan to read over the prior session's notes once a day, associating it with a standing, regular activity like a meal. Another possibility for augmenting Ethan's treatment might have been to provide a speech therapy referral, like that given to Alice, as both patients' speech deficits contributed to both their reticence in sessions and their broader struggles with self-confidence.

As Durland notes, addressing Fay's expressed hostility earlier in the treatment might have increased Ethan's likelihood of benefitting from the intervention. Initiating a conversation about a care-partner's problematic behavior, while simultaneously building an alliance with that person, is a daunting prospect for any clinician — particularly when the patient's treatment participation seems to depend on their partner's involvement. Durland carefully weighed his decisions about Fay's role in treatment, but one likely influence he does not explicitly mention is the in-session effect of Fay's hostility on Durland, and its potential contribution to his relatively acquiescent approach. To utilize Durland's behavioral observations toward preserving rapport with Fay, an individual conversation with her might have taken as a starting point the real challenge she faces in constant, high-demand caregiving. In this context, Durland could have explained his interest in holding some independent sessions with Ethan to assess his capabilities as benefitting Fay, and their relationship as well, by giving her a brief weekly break and seeking possibilities for some small, incremental changes to the dependent dynamic that so overtaxed Fay in other domains.

As Durland describes, Interpersonal Effectiveness skills presented to both partners, after a conflict between them late in treatment necessitates an individual conversation with Fay, are not especially accessible to Ethan. However, as Fay employs the skills, and concurrently allows Ethan more leeway to self-manage PD and household chores, positive things happen: they reportedly have fewer arguments; he shows some self-management capabilities that surprise her; and his confidence begins to improve. In retrospect, these developments suggest that Fay's hostile behavior toward Ethan in sessions may have reflected feeling overwhelmed by and resentful of over-functioning as a caregiver - as she appeared to do in some respects during their sessions - in addition to, as Durland explains, lacking sufficient understanding of Ethan's cognitive deficits.

\section{CARL, A RESPONDER, AND DORIS: INVESTIGATING PRESENT-MOMENT EXPERIENCE IN TANGENTIALITY}

Cognitive difficulties also played a significant role in Carl's treatment, though he presented as significantly less impaired than Ethan. In Carl's case, two interrelated challenges significantly affected his behavior in sessions and ability to engage with the protocol. For one, his worldview diverged significantly from the program material, which he found "dry, stuffy, and over-reliant on rationality" (p. 83). Related to this mismatch, Carl also told tangential, elaborate stories during sessions, resisting Durland's redirection by talking over him and returning repeatedly to his topic of choice. Durland describes working most effectively with Carl 
Individualizing Evidence-Based Treatment of Neuropsychiatrically Complex Patients: Process-Based Targets For Change in Parkinson's Disease

S.L. Mann, R. Miller, L. St. Hill, \& R.D. Dobkin

Pragmatic Case Studies in Psychotherapy, http://pcsp.libraries.rutgers.edu

Volume 16, Module 1, Article 2, pp. 104-117, 07-25-20 [copyright by authors]

when he tied the content of Carl's tangents to program-relevant topics, though devising these connections and harnessing Carl's interest on the spur of the moment was difficult.

Durland's challenge, then, takes the form of an amicable struggle with Carl for control over the session content. That is, Durland's concerns about treatment adherence conflict with Carl's persistent tangentiality. In addressing this pattern, one possibility not directly addressed is that it potentially reflects executive dysfunction, which is common in PD and exacerbated by depression (Dirnberger \& Jahanshahi, 2013; Lockwood, Alexopoulos, \& van Gorp, 2002), including impaired inhibitory control (spurring elaborations on anything of interest) and set shifting (perseverating when Durland attempts redirection). Carl nonetheless presented as eager to connect and share his experiences. Furthermore, he was willing and able to engage with program concepts when he connected them closely to his own words and views. In these instances, Durland was able to align with Carl to focus on a shared, often metaphorical problem conceptualization (e.g., "the depression genie"), which enlivened protocol concepts and drew on Carl's interests.

Carl's responsiveness to connecting content from his tangents to program material suggests that drawing on Carl's in-session experiences with storytelling during sessions might also have helped him to absorb some program concepts. Exploring, for example, how Carl felt in the moment as he shared fond memories of "running five miles a day" might have helped demonstrate the connections in the CBT model linking emotions to both thoughts (emotions evoked by the fond memory itself) and behaviors (feelings while sharing a cherished story). In this respect, rather than asserting the priority of program content, per se, inquiring about how Carl felt while telling the story, and how those observations might reflect the CBT model, might have provided Durland with a sincere, patient-centered, and program-relevant way to draw the treatment content into Carl's areas of focused interest. With this approach, Carl's tangential storytelling might have provided an illustrative behavior sample, rich with real-time cognitive and emotional associations to discuss, rather than a deviation that competes with a manualized lesson.

Furthermore, highlighting present-moment connections linking behavior, thought, and emotions might have served as helpful rehearsals for Carl's thought log homework assignments. Modifying these assignments to provide extended practice with identifying and naming connected behaviors-thoughts-emotion connections across a variety of situations might have provided a way to probe the CBT model's flexibility with Carl, while also providing Durland a clearer view of Carl's own unique construal of these relationships. While developing this understanding would likely require covering fewer skills in the course of treatment, it might have strengthened their alliance and developed a shared foundation (by returning to Carl's real-time self-observations) that could assist Carl in learning and practicing other skills.

Approaching certain program material via Carl's in-situ experience also may have skirted some of his objections to aspects of the CBT model. Carl expressed strong beliefs that "emotions or physical sensations precede thought" and that "reason' was incapable of exerting control over emotions." Though these views contrast with the premise of cognitive restructuring, a skill 
Individualizing Evidence-Based Treatment of Neuropsychiatrically Complex

Patients: Process-Based Targets For Change in Parkinson's Disease

S.L. Mann, R. Miller, L. St. Hill, \& R.D. Dobkin

Pragmatic Case Studies in Psychotherapy, http://pcsp.libraries.rutgers.edu

Volume 16, Module 1, Article 2, pp. 104-117, 07-25-20 [copyright by authors]

emphasized in the protocol, they also demonstrate Carl's awareness of, and interest in, the very aspects of his experience that serve as building blocks for CBT skills: thoughts, behaviors, physical sensations, emotions, and their relationships. The CBT model, as presented early in the protocol, emphasizes interconnections among these factors rather than specifying a unidirectional causal chain, in which, for example, thoughts necessarily precede and effect emotions. Thus, one of the protocol's key messages about the CBT model is that several connected factors work together to influence mood - offering various points (cognitive, behavioral, biological) for intervention. One approach would have been (a) to help Carl to observe and explore these connections in sessions as he experienced them; (b) to record these observations in session on a simplified thought log for practice; and then (c) to modify homework to consolidate and generalize this self-observation skill. This approach might have provided the pair with additional discussion points based on Carl's own interests and experiences.

In addition, changing the order of the modules to front-load program skills potentially suited to Carl's experience and interests might have strengthened the alliance earlier in treatment and provided positive experiences to reinforce willingness to try out additional skills. Given his interests and beliefs, as well as strong emotional connections to certain positive memories, Carl might have benefitted from learning and practicing relaxation techniques like guided imagery in earlier sessions. This practice also might have provided another opportunity to focus the conversation on Carl's in-situ experience and self-observation, in support of developing new a coping skill.

\section{GARY, A NONRESPONDER: DISENGAGEMENT AS A DISTRESS SIGNAL}

Gary presented similar, if more profound, challenges than Carl related to his negative reactions to the program content and structure. From the start, he challenged every aspect of the treatment, from questioning Durland's clinical competence to dismissing the program materials and rejecting even the prospect of scheduling sessions as "too stressful." He asks instead that Durland "call him whenever [Durland ] was free, and should [Gary] also be free, [they] could have the session." This preference presents a major obstacle to consistent, quality care by rendering session frequency and length unpredictable and compromising both participants' ability to prepare.

Nonetheless, Durland works to address Gary's expressed needs by not only meeting this request but persisting with treatment even as Gary is "frequently distracted or involved in other activities" (p. 78) during sessions. Ironically, Durland's diligence in trying to push past these barriers and remain protocol-adherent may have contributed to leaving some of Gary's significant presenting problems unaddressed - because, in Gary's case, these difficulties manifested in his stance toward treatment itself, rather than in response to particular program content. This becomes clear in Gary's exit interview, in which he insightfully reflects on his disengaged, critical stance toward the treatment, linking it to his nonacceptance, at that time, of 
Individualizing Evidence-Based Treatment of Neuropsychiatrically Complex

Patients: Process-Based Targets For Change in Parkinson's Disease

S.L. Mann, R. Miller, L. St. Hill, \& R.D. Dobkin

Pragmatic Case Studies in Psychotherapy, http://pcsp.libraries.rutgers.edu

Volume 16, Module 1, Article 2, pp. 104-117, 07-25-20 [copyright by authors]

his PD diagnosis and the pressure he felt to "accomplish [everything] he hoped to achieve in life before his symptoms left him incapacitated" (p. 78).

It remains unclear how aware Gary may have been of these insights during the treatment. But Durland's description of him "repeatedly mention[ing] feeling pressed for time and 'all over the place"” (p. 78) suggests he was, at minimum, aware of painful, pervasive, diagnosis-related anxiety and worries which, if approached directly, might have been drawn out and addressed. Gary's comments about stress and time pressure appear directly connected to his refusal to schedule appointments and his insistence on multitasking during session calls. These, in turn, reflect the anxiety and despair about his diagnosis that led to his frantic attempts to expedite a lifetime of productivity. In that context, thoughtful, focused psychotherapy for the depression likely underlying his anxiety becomes all but impossible.

Given the time-consuming treatment trial enrollment process, Durland could presume that despite the urgency Gary felt in many areas of his life, Gary had expended significant effort to access this form of treatment at this time, only to withdraw when it became available. This behavior suggested that Gary's attitude toward treatment was more complex and ambivalent than his consistently dismissive, critical comments indicate, perhaps taking the form of an approachavoidance conflict. With this in mind, it might have been helpful to address Gary's skeptical stance and distancing behavior directly although gently, as soon as these patterns began to define his relationship to Durland and to the treatment process. The aim of these conversations might have been to air and acknowledge Gary's hesitations about treatment, while also inquiring about the motivations that drew him to enroll in the trial at this time.

Adopting a curious, open, and receptive position with regard to Gary's pushback against elements of the program, along the lines of "rolling with resistance" in the Motivational Interviewing approach Durland later considers (Miller \& Rollnick, 2012), might have tangibly demonstrated to Gary the program's collaborative approach and Durland's interest in understanding Gary's individual experience. In these respects, this approach might have drawn a contrast between the developing alliance and Gary's reportedly disappointing, somewhat adversarial relationships with other providers. In retrospect, Gary's "stated desire that [they] 'just talk, and [Durland's] sense of responsibility to implement the treatment as intended" (p. 79) might have been bridged by agreeing to briefly shelve the protocol, "just talk" for a session —and negotiate the inclusion of some specific, treatment-relevant topics. For example, Durland might have used this less-structured session to elicit more information about Gary's understanding of his depression, including its causes and consequences, and his concerns about starting psychotherapy (e.g., feeling time-constrained and disorganized, doubting the manualized materials).

This understanding might have provided a shared rationale for some preliminary, collaborative steps toward effective intervention. Such steps might have included agreeing to schedule only the subsequent session in advance, for now, and to spend some time in that session considering whether the cognitive behavior therapy (CBT) model might offer another way of looking at Gary's experiences. It would be important to clarify in these conversations that 
Individualizing Evidence-Based Treatment of Neuropsychiatrically Complex

Patients: Process-Based Targets For Change in Parkinson's Disease

S.L. Mann, R. Miller, L. St. Hill, \& R.D. Dobkin

Pragmatic Case Studies in Psychotherapy, http://pcsp.libraries.rutgers.edu

Volume 16, Module 1, Article 2, pp. 104-117, 07-25-20 [copyright by authors]

committing to the next appointment time or "trying on" the CBT model would not require abrupt changes from Gary, but would depend on his honest input, a critical component of this treatment approach.

Slowing the pace at first, while inviting Gary to reflect openly on the CBT model, rather than teaching it as a lesson, might have effectively set up an in-session behavioral experiment, without the need to frame it as such. That is, Gary's in-session experiences would take on a more important role initially than his responses to the agreement to schedule or the CBT model. It's true that Gary could have provided negative feedback about his willingness to collaborate about scheduling (behavior change); his willingness to consider a new framework for understanding his mood (cognitive change); and (3) his willingness to discuss his experiences with these changes. Still, this negative feedback might have provided Durland a "foot in the door" toward aligning with Gary earlier in treatment. From here, depending on Gary's needs, preferences, and willingness to engage (e.g., complete homework), treatment adaptations might have included sampling from the manual to teach Gary a smaller, personalized subset of coping skills, or adopting a more principles-based CBT approach, referencing manualized readings and skills only as relevant.

\section{CONCLUSION}

In considering the need to individually tailor evidence-based treatments, it is important to consider a key obstacle to such adaptations, which Durland highlights in Gary's case and confronts in the other cases as well. As a trainee, Durland provided this treatment within a randomized, controlled trial, and he recalls being uncertain of "the amount and types of modifications allowed for by the program" (p. 79), as well as believing his competence would be judged by his protocol fidelity. As Durland later realized, limiting his communication with his supervisor about the difficulty of engaging Gary in the protocol limited, in turn, his communication with Gary himself, which was constrained by Durland's belief that adherence needed to take precedence over flexibility. Durland is candid and reflective about questioning the extent to which protocol deviations might be helpful or detrimental (both to Gary's treatment and, as a potentially separate matter, to the RCT data quality), connecting his uncertainty with his trainee status when he suggests it might have been remedied by "asking for help." While supervisory guidance might have helped Durland to proceed differently with Gary, his uncertainty about the nature and magnitude of effective protocol flexibility reflects a core issue in evidence-based practice research and implementation.

Though it is by definition difficult to systematize and measure individualized adaptations to treatment protocols, the literature indicates that adaptations have been especially likely to improve outcomes when they are "discrete, well-defined, and based on sound theory and an understanding of the population"(Wiltsey Stirman, Gamarra, Bartlett, Calloway, \& Gutner, 2017). As a lens for applying behaviorist theory to the processes and dynamics that unfold during sessions, the functional analytic psychotherapy (FAP) approach can highlight process-based therapeutic targets, which, by virtue of happening in real-time therapist-patient interactions, may prove especially accessible for collaborative observation and rapidly reinforced change. It can 
Individualizing Evidence-Based Treatment of Neuropsychiatrically Complex

Patients: Process-Based Targets For Change in Parkinson's Disease

S.L. Mann, R. Miller, L. St. Hill, \& R.D. Dobkin

Pragmatic Case Studies in Psychotherapy, http://pcsp.libraries.rutgers.edu

Volume 16, Module 1, Article 2, pp. 104-117, 07-25-20 [copyright by authors]

also point clinicians toward incorporating into the treatment other evidence-based techniques (e.g., mindful, present-moment awareness and motivational interviewing) in order to understand a patient's experience in the course of struggling with or resisting a particular coping skill, and to therapeutically address the causes of difficulty.

As these cases demonstrate, combining this perspective with an understanding of PDspecific challenges and limitations (e.g., symptom-related shame and social withdrawal, and common manifestations of PD executive dysfunction) can equip clinicians to bridge the inevitable gaps between the strengths, preferences, and functional limitations of individual patients and the protocol as written. For Alice, this meant not only using behavioral activation goals to increase her participation in sessions, but also to collaboratively create a supportive, responsive in-session environment in which Durland and Bob positively reinforced Alice's efforts. This change allowed Alice to engage more fully in the rest of her treatment, and the process modeled a new problem-solving approach that the couple could apply as they made other behavioral changes, with Alice leading the way.

While Fay helped bridge communication and comprehension gaps for Ethan, she brought a punishing tone to her intermediary role. Viewing this dynamic as a reprise of depressionmaintaining relationship patterns might have helped Durland to tailor treatment priorities more closely to Ethan's needs. Rather than hewing toward adherence, which depended on Fay, Durland might have suggested individual sessions with each party, in which to assess Ethan's independent functioning and to adapt the protocol accordingly, while directly addressing Fay's communication style.

Carl's disagreements with certain protocol concepts and compelling interests in tangential topics made it challenging to engage him in skills development for most of the treatment course. He was, however, eager to connect by sharing emotionally resonant stories, which might have provided better opportunities to discuss basics of the CBT model-i.e., connections between his emotions, thoughts, and behaviors - by eliciting observations of his in-session experiences.

Finally, Gary takes an adverse, noncommittal, approach to treatment, impeding his engagement with the protocol-yet, his exit interview reveals that his harried disengagement reflected overriding anxiety and nonacceptance of his PD diagnosis. In retrospect, directly addressing Gary's in-session avoidance through adaptations like motivational interviewing or compromising aspects of treatment structure might have strengthened the alliance between Gary and therapist Durland and helped draw these issues to the surface.

Durland's sensitive treatment adaptations for these patients, and the additional opportunities for flexible tailoring discussed above, align with a focus on the individual patient's treatment experience in process-based approaches to CBT currently gaining prominence (Hayes et al., 2019). These approaches recognize that coping skills-based treatments not only impart new skills; they also provide supportive training in the challenging, often uncomfortable, and necessarily experiential process of learning how to learn such skills - that is, learning how to change. Marrying the strengths of coping-skills based, empirically supported treatments with systematic attention to these process-based factors can help patients develop the flexibility and 
Individualizing Evidence-Based Treatment of Neuropsychiatrically Complex Patients: Process-Based Targets For Change in Parkinson's Disease

S.L. Mann, R. Miller, L. St. Hill, \& R.D. Dobkin

Pragmatic Case Studies in Psychotherapy, http://pcsp.libraries.rutgers.edu

Volume 16, Module 1, Article 2, pp. 104-117, 07-25-20 [copyright by authors]

self-efficacy to generalize new skills effectively to novel situations. As people managing depression and/or anxiety as well as Parkinson's disease or other functionally limiting chronic health problems face daily demands for problem-solving around unpredictable symptom fluctuations and illness-related constraints (Bodenheimer, Lorig, Holman, \& Grumbach, 2002) this ability can make the difference between optimal functioning and steep decline. For clinicians, therefore, a key principle of balancing protocol fidelity with flexibility for these patients must involve attending to both the content and the process of skill development.

\section{REFERENCES}

American Psychological Association, (2005). Report of the 2005 presidential task force on evidence-based practice. Washington, DC: Author. https://www.apa.org/practice/resources/evidence/evidence-based-report.pdf

Bodenheimer, T., Lorig, K., Holman, H., \& Grumbach, K. (2002). Patient self-management of chronic disease in primary care. JAMA, 288(19), 2469-2475.

Dirnberger, G., \& Jahanshahi, M. (2013). Executive dysfunction in P arkinson's disease: A review. Journal of Neuropsychology, 7(2), 193-224.

Dobkin, R. D., Mann, S. L., Gara, M. A., Interian, A., Rodriguez, K. M., \& Menza, M. (2020a, April 21). Telephone-based cognitive behavioral therapy for depression in Parkinson disease. A randomized controlled trial. Neurology, 94 (116). doi: 10.1212/wnl.0000000000009292

Dobkin, R. D., Mann, S. L., St. Hill, L., Rodriguez, K. M., Miller, R., King, A. R., . . Interian, A. (2020b). Video-to-home cognitive-behavioral therapy for veterans with Parkinson's disease and depression: A randomized controlled trial. International Congress of Parkinson's Disease and Movement Disorders; September 13, 2020; Philadelphia, PA. .

Durland, L. (2020). Telephone-based, clinician-guided self-help cognitive behavioral therapy for depression in Parkinson's disease (dPD): The responder cases of "Alice" and "Carl," and the nonresponder cases of "Ethan" and "Gary." Pragmatic Case Studies in Psychotherapy, 16(1), Article 1, 1-103. Available: http://pcsp.libraries.rutgers.edu/

Forsaa, E. B., Larsen, J. P., Wentzel-Larsen, T., Herlofson, K., \& Alves, G. (2008). Predictors and course of health-related quality of life in Parkinson's disease. Movement Disorders, 23(10), 1420-1427. doi:10.1002/mds.22121

Hayes, S. C., Hofmann, S. G., Stanton, C. E., Carpenter, J. K., Sanford, B. T., Curtiss, J. E., \& Ciarrochi, J. (2019). The role of the individual in the coming era of process-based therapy. Behaviour Research and Therapy, 117, 40-53.

Hinnell, C., Hurt, C. S., Landau, S., Brown, R. G., Samuel, M., \& Group, P. P. S. (2012). Nonmotor versus motor symptoms: how much do they matter to health status in Parkinson's disease? Movement Disorders, 27(2), 236-241.

Holman, G., Kohlenberg, R. J., Tsai, M., Haworth, K., Jacobson, E., \& Liu, S. (2012). Functional Analytic Psychotherapy is a framework for implementing evidence-based practices: The example of integrated smoking cessation and depression treatment. International journal of behavioral consultation and therapy, 7(2-3), 58. 
Individualizing Evidence-Based Treatment of Neuropsychiatrically Complex Patients: Process-Based Targets For Change in Parkinson's Disease

S.L. Mann, R. Miller, L. St. Hill, \& R.D. Dobkin

Pragmatic Case Studies in Psychotherapy, http://pcsp.libraries.rutgers.edu

Volume 16, Module 1, Article 2, pp. 104-117, 07-25-20 [copyright by authors]

Hopko, D. R., \& Hopko, S. D. (1999). What can functional analytic psychotherapy contribute to empirically-validated treatments? Clinical Psychology \& Psychotherapy: An International Journal of Theory \& Practice, 6(5), 349-356.

Kanter, J. W., Manbeck, K. E., Kuczynski, A. M., Maitland, D. W., Villas-Bôas, A., \& Ortega, M. A. R. (2017). A comprehensive review of research on functional analytic psychotherapy. Clinical Psychology Review, 58, 141-156.

Laska, K. M., Gurman, A. S., \& Wampold, B. E. (2014). Expanding the lens of evidence-based practice in psychotherapy: A common factors perspective. Psychotherapy, 51(4), 467.

Lockwood, K. A., Alexopoulos, G. S., \& van Gorp, W. G. (2002). Executive dysfunction in geriatric depression. American Journal of Psychiatry, 159(7), 1119-1126.

Martínez-Martín, P., Forjaz, M. J., Frades-Payo, B., Rusinol, A. B., Fernández-García, J. M., Benito-León, J., . . Catalán, M. J. (2007). Caregiver burden in Parkinson's disease. Movement Disorders, 22(7), 924-931.

Miller, W. R., \& Rollnick, S. (2012). Motivational interviewing: Helping people change: Guilford press.

Pontone, G. M., Dissanayaka, N., Dobkin, R. D., Mari, L., Marsh, L., Vernaleo, B. A., .. . Mari, Z. (2019). Integration and extension of specialty mental healthcare services to community practice in Parkinson's disease. The American Journal of Geriatric Psychiatry.

Titova, N., \& Chaudhuri, K. R. (2017). Personalized medicine in Parkinson's disease: time to be precise. Movement Disorders, 32(8), 1147.

Weintraub, D., \& Mamikonyan, E. (2019). The neuropsychiatry of Parkinson disease: a perfect storm. The American Journal of Geriatric Psychiatry.

Wiltsey Stirman, S., Gamarra, J. M., Bartlett, B. A., Calloway, A., \& Gutner, C. A. (2017). Empirical examinations of modifications and adaptations to evidence-based psychotherapies: Methodologies, impact, and future directions. Clinical Psychology: Science and Practice, 24(4), 396-420. 